\title{
Editorial
}

\section{La competencia profesional clínica del especialista en medicina familiar}

\author{
Professional Clinic Competence of the Family Medicine Specialist \\ A Competência Profissional Clínica do especialista em medicina de família
}

Francisco Javier F. Gómez-Clavelina, Isaías Hernández-Torres," María Guadalupe Grijalva

La competencia ha sido definida como un constructo complejo, multifacético, multivariado, multidimensional, a menudo en relación con una situación multidisciplinaria, en particular en el campo de la medicina. A este concepto se ha incorporado el término "profesional" definiéndola como "el uso habitual y juicioso de la comunicación, el conocimiento, las habilidades técnicas, razonamiento clínico, emociones, valores y reflexión en la práctica diaria para el beneficio del individuo y de la comunidad a la que sirve". ${ }^{2}$ Según Norman, la competencia clínica es un conjunto de atributos multidimensionales y hace la siguiente categorización:

- Habilidades clínicas: capacidad para obtener información al interrogar y examinar pacientes e interpretar su significado

- Conocimientos y comprensión: habilidad para recordar conocimientos relevantes acerca de condiciones clínicas que lleven a proveer atención médica efectiva y eficiente para los pacientes

- Atributos interpersonales: expresión de aquellos aspectos de carácter profesional del médico que son observables en las interacciones con pacientes
- Solución de problemas y juicio clínico: aplicación del conocimiento relevante, habilidades clínicas y atributos interpersonales para el diagnóstico, investigación y manejo de los problemas de un paciente dado

- Habilidades técnicas: habilidad para usar procedimientos y técnicas especiales en la investigación y manejo de pacientes ${ }^{3}$

La competencia se construye fundamentándose en habilidades básicas clínicas, conocimientos científicos y el desarrollo moral. Incluye una función cognitiva que consiste en adquirir y utilizar el conocimiento para resolver problemas de la vida real; una función integradora, utilizando datos biomédicos y psicosociales en el razonamiento clínico; una función relacional que incluye la comunicación efectiva tanto con los pacientes como con los colegas; y una función afectiva-moral que incluye la voluntad, la paciencia y la inteligencia emocional para aplicar estas habilidades con prudencia y humanidad. ${ }^{2}$

Con base en estas consideraciones y los principios de la medicina familiar (atención médica integral, enfoque de riesgo y continuidad de la atención), definimos la competencia profesional clínica del especialista en medicina familiar como: el conjunto de conocimientos, habilidades, actitudes y valores que, basados en el razonamiento clínico, juicio crítico acertado y de conformidad con el estado actual del avance científico, el médico familiar aplica para tener un desempeño profesional eficiente en la solución de los problemas de salud

Profesores de la Subdivisión de Medicina Familiar de la Direc-

Este artículo debe citarse: Gómez-Clavelina FJF, Hernández-Torres I, Grijalva MG. ción de Estudios de Posgrado La competencia profesional clínica del especialista en medicina familiar. Aten Fam. de la Universidad Nacional 2014; 21(4):101-102. 
individuales y colectivos de la sociedad, en un contexto específico.

Este constructo lleva implícito un aspecto de dinamismo, que está constituido por dos elementos fundamentales: el contexto y la selectividad. El contexto se refiere al escenario clínico en el cual se desarrolla la competencia; la selectividad se refiere a la capacidad de priorizar la dimensión biológica, psicológica o social de los problemas de salud. ${ }^{4}$

En el ámbito internacional, los programas para la formación de médicos especialistas están considerando su refuerzo mediante el desarrollo de competencias en seis dominios, recopilando información del desempeño que, en forma confiable y precisa, representa la capacidad de los residentes para atender a sus pacientes y trabajar en forma efectiva en los sistemas de otorgamiento de la atención sanitaria. Cuando los médicos adquieren y aplican las competencias en forma efectiva, se asume que el resultado es la calidad en la atención al paciente. ${ }^{5}$

La educación basada en competencias en los profesionales de la salud se ha constituido en un enfoque de gran relevancia en el ámbito de los posgrados en Canadá, Países Bajos, Reino Unido, Estados Unidos de Norteamérica y en muchos otros países. Los marcos de referencia o áreas de competencia, que se han desarrollado a nivel nacional e internacional han sido bien recibidos y en muchas ocasiones los gobiernos los han considerado obligatorios. ${ }^{6}$

Con fundamento en el Plan Nacional de Desarrollo 2013-2018, que considera necesaria la aplicación de estrategias para mejorar la calidad en la formación de recursos humanos en el ámbito de la salud; el Plan de Desarrollo de la Universidad Nacional Autónoma de México (UNAM); así como el Plan de Desarrollo de la Facultad de Medicina, en la División de Estudios de Posgrado (DEP), se estableció el objetivo de modernizar y optimizar los programas de especialidades, su método de enseñanza y evaluación, basados en competencias para mejorar las destrezas médico quirúrgicas, así como la calidad de la educación y la atención médica. La Subdivisión de Medicina Familiar de la DEP, está desarrollando un proyecto educativo dirigido a la identificación, enseñanza y evaluación de las competencias en medicina familiar. ${ }^{7-9}$

\section{Referencias}

1. Brailovsky CA. Educación médica, evaluación de las competencias. En: ops/oms, editores. Aportes para un cambio curricular en Argentina. Buenos Aires: University Press; 2001. 103-20.

2. Epstein RM, Hundert EM. Professional Competence. JAMA. 2002;287(2):226-35.

3. Norman G. Defining Competence: A Methodological Review. En: Neufeld VR, Norman GR, editores. Assessing Clinical Competence. New York: Springer Publishing Company; 1985. 15-37.

4. Abreu HLF, Cid GAN, Herrera CG, Lara VJVM, Laviada DR, Rodríguez ACSA. Perfil por Competencias del Médico General Mexicano. México: Asociación Mexicana de Facultades y Escuelas de Medicina (AMFEM), Elsevier Masson Doyma; 2008. 1-39.

5. Swing SR. The ACGME outcome project: retrospective and prospective. Med. Teach. [Internet]. 2007 Sep;29(7):648-54. [Citado 2013 Sep 16] Disponible en: http://www.ncbi.nlm.nih.gov/ pubmed/18236251

6. Ten Cate O, Snell L, Carraccio C. Medical competence: the interplay between individual ability and the health care environment. Med. Teach. [Internet]. 2010 Jan;32(8):669-75. [Citado 2013 Sep 30]. Disponible en: http://www.ncbi.nlm.nih.gov/ pubmed/20662579

7. Gobierno de la República, Estados Unidos Mexicanos. Plan Nacional de Desarrollo 2013-2018. Estrategia 2.3.4 Líneas de acción 3 y 4. México; 2013. 117.

8. Junta de Gobierno de la UNAM. Plan de Desarrollo de la UNAM 2011-2015. Universidad Nacional Autónoma de México; 2011. 13.

9. Facultad de Medicina, Universidad Nacional Autónoma de México. Plan de Desarrollo de la Facultad de Medicina 2012-2016. unAm; 2011. 18. 